\title{
Numerical Investigation on Magnetically Actuated Tunable Micro Liquid Lens
}

\author{
Shi-Hua $\mathrm{Wu}^{1}$, Gui-Ping Zhu ${ }^{1 *}$, and Hu-Lin Huang ${ }^{1}$ \\ ${ }^{1}$ College of Astronautics, Nanjing University of Aeronautics and Astronautics, Nanjing 210016, China
}

\begin{abstract}
In this paper, magnetically actuated tunable liquid lens is fundamentally studied to have further potential application scope in research, industrial, aeronautical and astronautical areas. The magnetic field, which is generated by a magnetic potential applied to the permanent magnet domain, distributes non-uniformly in the computational domain and generates magnetic field force to obtain the deformation of the ferrofluid droplet. Consequently, the light-transmitting droplet deforms due to direct contact with the ferrofluid droplet by a connecting channel. The combined effects of gravitational, frictional, interfacial and pressure-driven force make light-transmissive droplet to be a programmable liquid lens. The light-transmissive droplet exhibits changes in the curvature of the contour, which results in different focal lengths for realizing the variable-focus liquid lens. This work focus on the driven mechanism by the magnetic force for a better understanding. The performance is numerically studied for a magnetically actuated varifocal liquid lens by finite element method. The results are presented individually for the distribution of magnetic and flow field, and light refraction.
\end{abstract}

\section{Introduction}

In the recent decade, with the demand for various digital products such as smart phones, digital cameras, surveillance cameras, car cameras and microscopes, zoomable liquid lenses have received increasing attention in the field of imaging. Conventional imaging devices are usually composed of several convex lenses. For example, the microscope adjusts the focus by adjusting mechanical threads between the convex lenses. Such operation is very complicated, and the device is bulky, cumbersome and expensive. Conventional glass or resin convex lenses require high-precision machine tools to manufacture with high cost. The liquid lens is made of light-transmissive liquid with no mechanical processing and low cost of manufacturing. The liquid lens, which maintains its surface profile by gravity and interfacial tension, does not break under severe vibration. Moreover, the surface can be self-repairing and very smooth. Liquid lens requires only one lens to achieve zoom without polarization in the imaging.

With the development of digital microfluidics and micromechanical electronics, there have been many ways developed to control the zoom lens. The most common way is the fluid pressure actuation [1-5], which adjusts the focal length of the light-transmitting liquid by the movement of the other fluid. The other approaches for tuning the focal length of the liquid lens includes electrowetting [6-12], dielectrophoresis [13-15] and thermal control [16]. Some liquid lenses are produced by filling a thin film material with a light-transmitting liquid to prevent evaporation of the liquid [17-21]. All the controlling methods adjust the focal length of the liquid lens by changing the curvature of the liquid contour. Fluid pressure actuation methods typically require complex mechanical actuation devices that are more sensitive to vibration than electrowetting and dielectrophoresis methods. It is not suitable for applying fluid pressure actuation in high speed imaging due to a certain delay in zooming as an indirect drive method. The electrowetting and dielectrophoresis methods can operate directly on the light-transmissive liquid. By apply a high voltage on the fluid, the strong zooming ability exhibits with a relatively simple structure with no other mechanical actuating parts. However, the problem of liquid evaporation is required to be properly addressed.

The magnetic actuation [18,19,21-24] method attracts increasing attentions due to easy fabrication and integration. Fluid pressure is induced to adjust the focal length of the light-transmitting liquid. Magnetic actuation can be further divided into two categories that driven by ferrofluid and non-magnetic fluid. For example, the magnetic field generated by the electromagnetic coil was used to drive the movement of the ferrofluid to realize deformation of the liquid lens for zooming [22]. Malouin et al. [23] used ferrofluid droplets to act as liquid pistons with sinusoidal signals applied to the electromagnets to drive the liquid to zoom. Ferrofluids was actuated by electromagnets to drive

Corresponding author: zhuguiping@nuaa.edu.cn 
mechanically-wet liquid lens[24]. Oh et al. [21] used an electromagnet to drive the permanent magnet motion on the liquid and indirectly drive the liquid lens to achieve zooming. The reliability of magnetically actuated liquid lens is relatively high. However, there will be a delay of a millisecond for the deformation of the liquid lens responding to the change of the magnetic field.

So far as we know, most of work on magnetially driven liquid micro lens are realized by experimental

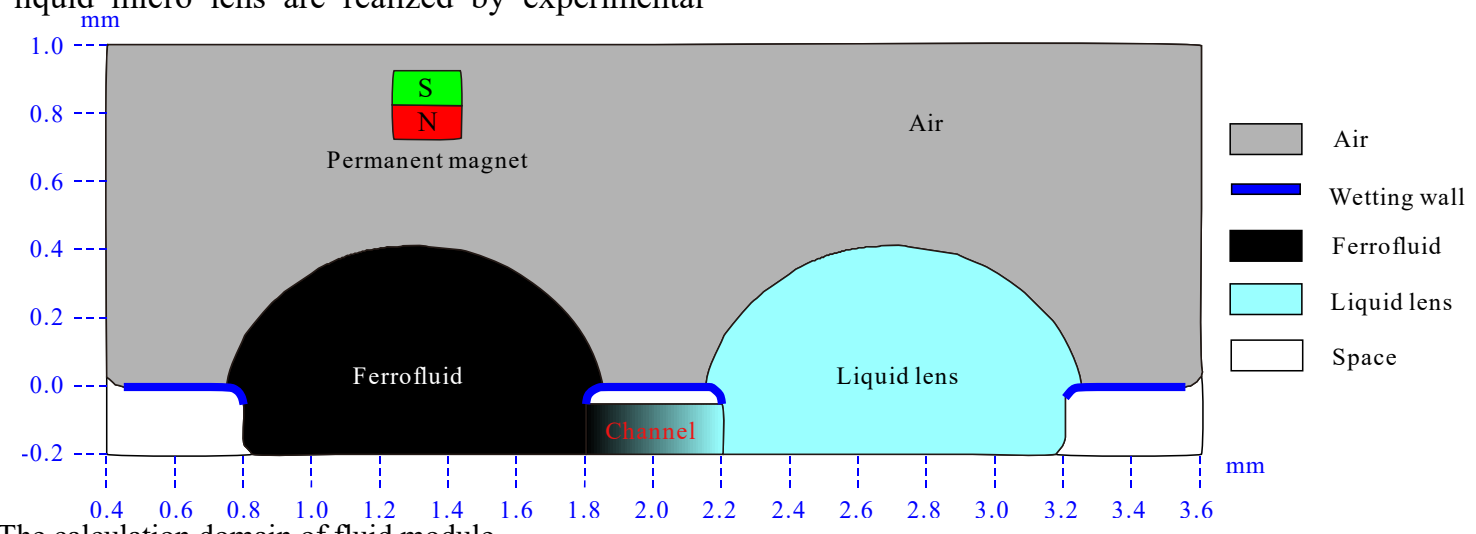

Fig. 1. The calculation domain of fluid module.
By using the finite element method, two-dimensional (2D) calculation is carried out to simulate a magnetically actuated microfluidic lens. The computational domain can be divided into fluid module, magnetic field module and optical module physically. The calculation domain of the fluid module is shown in Fig 1. Ferrofluid and liquid lens are represented by two semicircles approximately $1 \mathrm{~mm}$ in diameter. The fluid can flow in each other within two droplets connected by a square channel. The gray part is an air field with a density of 1.2 $\mathrm{kg} / \mathrm{m}^{3}$ and a dynamic viscosity of $18.1 \times 10^{-6} \mathrm{~Pa} \cdot \mathrm{s}$ as the second phase (continue phase, $\mathrm{CP}$ ) of the multiphase flow. As the first phase (Discrete phase, DP) of the multiphase flow, the ferrofluid and liquid lens are treated as the same medium with a uniform density of $1 \times 10^{3}$ $\mathrm{kg} / \mathrm{m}^{3}$ and a dynamic viscosity of $5 \times 10^{-3} \mathrm{~Pa} \cdot \mathrm{s}$. The two-phase flow of air and liquid is calculated by the level set module (LS) for tracking interface. The contact surfaces on both sides of the droplet are set to a wetted wall with a contact angle of 90 degrees. The tension between the droplet and air is set at $0.01 \mathrm{~N} / \mathrm{m}$. The space occupied by the permanent magnet and the blank space do not participate in the calculation. All the boundaries of the fluid domain are set as the outlet for eliminating the boundary effects.

The magnetic field currentless module (MFNC) is utilized for calcuting the magnetic field which only appears at the ferrofluid half region of the domain $(0.4 \sim 2.0 \mathrm{~mm})$ shown in Fig 1. Relative permeability of the permanent magnet domain is set at 5 . With the top edge set to zero, a magnetic potential of 4-12 $\mathrm{A}$ is applied to the base of the permanent magnet to generate a vertical upward magnetic field in the permanent magnet area. Regardless of saturation and residual magnetization, the relative magnetic permeability of the air and ferrofluid domain is set as 1 and 1.3, respectively. The computational domain employ the magnetic studies. For a better understanding of the physics and process, this work focused on the numerical calcualtion of magnetically driven lens at different field strength. In addition, the delay of deformaiton of the lens is considered in our discussion. The results are systematically presented in terms of the distribution of flow field, magnetic field and the optical properties. shielding boundaries for reduction of the boundary influence on the distribution of the magnetic field.

\section{Model and methods}

The optical performance is calculated by the geometric optics module (GOP), with the calculation domain shown in Fig 2. The semicircle with a diameter of about $1 \mathrm{~mm}$ is a liquid lens and the rest is air. The refractive index of the lens and air is respectively set to 1.5 and 1 without considering the light reflection. The refraction boundary of the droplet is highlighted in which the arc profile is redrawn into the optical module as a parametric curve by the coordinates determined when the level set function value is 0.5 . The light source release boundary on the top of the domain can uniformly emit visible light with a wavelength of $660 \mathrm{~nm}$ in the vertical downward direction (y-axis negative direction). The rest are the wall freezing boundaries that the light will stop automatically when passing through.

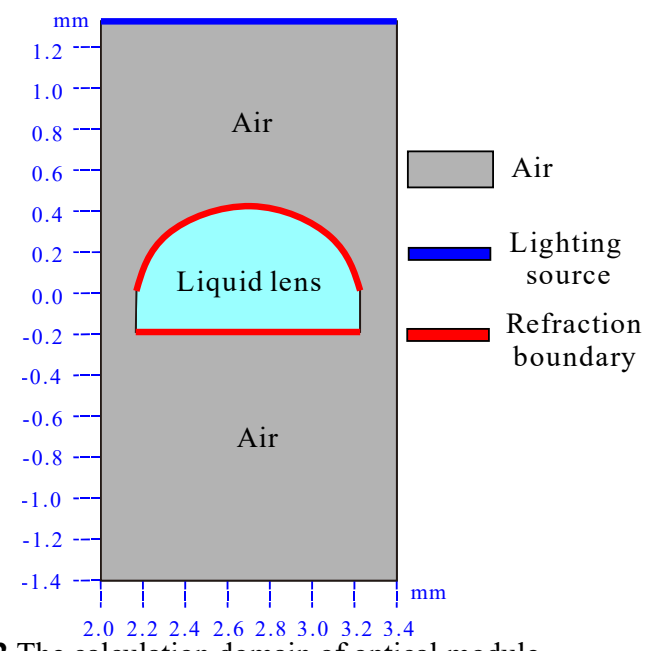

Fig. 2.The calculation domain of optical module. 
In the simulation, the optical part acts as an individual calculation module. The flow field and magnetic field simulation requires iterative calculation with MFNC, SPF and LS modules by the split solver. The detailed information of the calculation process is shown in Fig 3. Two cases are calculated with the toal mesh unit number at 14028 and 28698, respectively. The calculation is considered to be independent with mesh as the relative errors are less than $5 \%$ for various parameters such as the areal integral of magnetic force, maximum velocity, maximum pressure and so on.

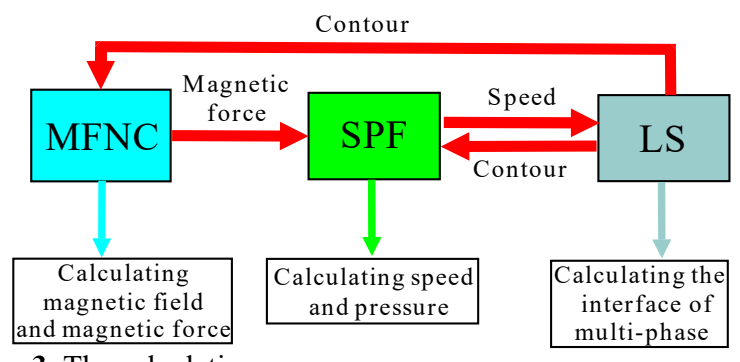

Fig. 3. The calculation process.

The magnetic field module is mainly used to calculate the magnetic field distribution without conduction current, which can be solved by the Maxwell's equations:

$$
\begin{gathered}
\nabla \cdot \mathbf{B}=0 \\
\nabla \times \mathbf{H}=0 \\
-\nabla V_{\mathrm{m}}=\mathbf{H} \\
\mathbf{B}=\mu_{0} \mu_{r} \mathbf{H}
\end{gathered}
$$

where $\mathbf{H}, \mathbf{B}, V_{\mathrm{m}}, \mu_{0}$ and $\mu_{r}$ are the magnetic field strength, magnetic flux density, magnetic potential, vacuum permeability and relative permeability. The electrodynamics gives the magnetic force to any medium:

$$
\mathbf{F}=\oiiint \oiiint_{V} \mathbf{J} \times \mathbf{B} d V
$$

where $\mathbf{J}$ is the current density and $V$ is the volume of the medium. For nonconducting ferrofluid, the magnetization current density $\mathbf{J}_{\mathbf{m}}$ can be expressed as:

$$
\mathbf{J}_{\mathbf{m}}=\nabla \times \mathbf{M}
$$

where $\mathrm{M}$ is magnetization, the magnetic force is derived as:

$$
\begin{gathered}
\mathbf{F}=\oiiint_{V}(\nabla \times \mathbf{M}) \times \mathbf{B} d V \\
\mathbf{M}=\chi \mathbf{H} \\
\chi=\mu_{r}-1 \\
\mu=\mu_{0} \mu_{r}
\end{gathered}
$$

where $\chi$ is the magnetic susceptibility. Regardless of the changes with the magnetic field, the magnetic permeability can be assigned to the fluid domain by the level set function:

$$
\mu=\mu_{0}\left(\left(\mu_{r}-1\right) \Phi+1\right)
$$

where $\Phi$ (Eq.(25)) is the level set function. The calculation with $\Phi$ equals 0 and 1 represents the permeability of vacuum and ferrofluid, respectively. The excessive permeability distribution is smoothed when $\Phi$ varies within 0 and 1 . As a result, the magnetic force acts only in the ferrofluid droplet domain in the form as:

$$
\mathbf{F}_{1}=\frac{1}{2} \mu_{0} \chi \oiiint_{V} \nabla \mathbf{H}^{2} d V
$$

The magnetic force in the ferrofluid droplet is a function of the magnetic susceptibility, the volume of the droplet and the magnitude of the magnetic intensity. In addition, the other component of the magnetic force is generated due to the magnetic permeability gradient:

$$
\mathbf{F}_{2}=-\frac{1}{2} \mu_{0} \mathbf{H}^{2} \oiiint_{V} \nabla \chi d V
$$

The applied external force in the calculation is expressed by the volume force:

$$
\mathbf{f}=\frac{d \mathbf{F}}{d \mathbf{V}}=\mathbf{f}_{1}+\mathbf{f}_{2}=\frac{1}{2} \mu_{0} \chi_{m} \nabla \mathbf{H}^{2}-\frac{1}{2} \mu_{0} \mathbf{H}^{2} \nabla \chi_{m}
$$

where $f_{1}$ and $f_{2}$ represent the volumetric forces of $F_{1}$ and $\mathrm{F}_{2}$. Since both ferrofluid and oil are incompressible fluids, which can be solve by N-S equations and continuous equations:

$$
\begin{gathered}
\rho \nabla \cdot \mathbf{u}=0 \\
\frac{\partial(\rho \mathbf{u})}{\partial t}+\mathbf{u} \cdot \nabla(\rho \mathbf{u})=-\nabla P+\mu \nabla \cdot\left(\nabla \mathbf{u}+\nabla \mathbf{u}^{T}\right)+\mathbf{f}^{\prime}
\end{gathered}
$$

where $\mathbf{u}$ is the velocity of the fluid, $P$ is the internal pressure, $\mu$ is the dynamic viscosity, $\rho$ is the fluid density, and $\mathbf{f}^{\prime}$ is volume force which include magnetic force, gravity and tension. The resistance of a spherical droplet in moving can be expressed as [25]:

$$
f_{d}=6 \pi r_{d} v_{c p} \eta_{c p}\left(1+2 \eta_{c p} / \eta_{d p}\right) /\left(1+\eta_{c p} / \eta_{d p}\right)
$$

where $r_{d}, v_{c p}, \eta_{c p}$, and $\eta_{d p}$ express the droplet radius, continuous phase velocity, continuous phase viscosity and separation phase viscosity, respectively. The unit area tension at the interface of the droplet can be expressed as [26]:

$$
\begin{gathered}
\mathbf{f}_{\sigma}=\sigma \delta \mathbf{k n} \\
\mathbf{\kappa}=-\nabla \cdot \mathbf{n}
\end{gathered}
$$




$$
\begin{gathered}
\mathbf{n}=\frac{\nabla \boldsymbol{\Phi}}{|\nabla \boldsymbol{\Phi}|} \\
\delta=|\Phi(1-\Phi)| \cdot|\nabla \Phi|
\end{gathered}
$$

where $\sigma$ is tension. $\delta$ is the Dirac function, which is only zero at the droplet interface and the boundary of fluid. $\boldsymbol{\kappa}$ is the interface curvature, $\mathbf{n}$ is the unit normal vector of the interface. The wetting wall solving equation:

$$
\begin{gathered}
\mathbf{n}_{w e t} \cdot \mathbf{u}=0 \\
\mathbf{f}_{w e t}=\sigma \delta\left(\mathbf{n}_{w e t} \cdot \mathbf{n}_{\mathrm{int}}-\cos \left(\theta_{w}\right)\right) \mathbf{n}_{\mathrm{int}}-\frac{\mu}{\beta} \mathbf{u} \\
\mathbf{n} \cdot\left(\varepsilon \nabla \Phi-\Phi(1-\Phi) \frac{\nabla \Phi}{|\nabla \Phi|}\right)
\end{gathered}
$$

whrer $\mathbf{n}_{\text {wet }}$ is the wall normal unit vector, $\mathbf{f}_{w e t}$ is the wall force, $\mathbf{n}_{\text {int }}$ is the unit vector of fluid motion direction, $\beta$ is the slip length which value is the mesh element size. The interface capture is accomplished by the level set method with governing equation as:

$$
\begin{gathered}
\frac{\partial \boldsymbol{\Phi}}{\partial t}+\nabla \cdot(\mathbf{u} \boldsymbol{\Phi})=\gamma \nabla \cdot\left(\varepsilon \nabla \boldsymbol{\Phi}-\boldsymbol{\Phi}(1-\boldsymbol{\Phi}) \frac{\nabla \boldsymbol{\Phi}}{|\nabla \boldsymbol{\Phi}|}\right) \\
\gamma=0.5+V_{m} \cdot 10^{-2}
\end{gathered}
$$

where $\gamma$ is the initialization parameter, indicating the maximum speed of the interface movement. $\varepsilon$ is the interface thickness control parameter, which is set to $40 \mu \mathrm{m}$. In this model, $\boldsymbol{\Phi}=0,1$ and 0.5 means ferrofluid, mineral oil and the boundary, respectively. The calculation of the optical part is mainly carried out by the Fresnel formula:

$$
\begin{gathered}
\mathbf{n}_{\mathbf{t}}=\eta \mathbf{n}_{\mathbf{i}}+\gamma \mathbf{n}_{\mathbf{s}} \\
\eta=\frac{n_{1}}{n_{2}} \\
\gamma=-\eta \cos \left(\theta_{i}\right)+\cos \left(\theta_{t}\right)
\end{gathered}
$$

where $\mathbf{n}_{\mathbf{t}}, \mathbf{n}_{\mathbf{i}}, \mathbf{n}_{\mathbf{s}}, \eta, \gamma, n_{1}, n_{2}, \theta_{i}$ and $\theta_{t}$ represent the refracted ray unit vector, the incident ray unit vector, the unit vector perpendicular to refractive surface, the refractive index, the auxiliary variable, the air refractive index, the lens refractive index, the incident angle and the refraction angle, respectively.

\section{Results analysis and discussion}

The magnetic flux density distribution is shown in Fig 4. The magnetic field of the permanent magnet domain can reach up to $0.7 \mathrm{~T}$. White lines with arrows indicates the distribution of the simulated magnetic field. For detailed information, the distribution of magnetic field is shown in Fig 4 (b) with focus on the flux density ranging between 0 and $20 \mathrm{mT}$. The magnetic flux density close to the ferrofluid region is only a few $\mathrm{mT}$.

The flow field distribution is shown in Fig 5. From Fig 5 (a), the internal velocity of the droplet does not reach equilibrium due to inertia at $\mathrm{t}=4 \mathrm{~ms}$. With interface moving up, the ferrofluid droplet exhibits obvious four velocity vortex mainly due to the effect of the magnetic force. The liquid in the middle channel moves correspondingly to the ferrofluid. With the interface moving down, only two velocity vortices appear at the droplet on the right side of the domain. The high velocity values are mainly concentrated on both sides of the droplet due to the effect of wetting wall. At $\mathrm{t}=100 \mathrm{~ms}$ shown in Fig 5 (b), the steady state of the droplet is achieved under the stabilized flow field with no obvious movement of the droplet interface and basical disappearance of the velocity vortex. The larger velocity value mainly concentrates near the interface of the droplet due to the effect of surface tension. The corresponding pressure distribution is shown in Fig 5 (c). The larger pressure value mainly concentrates near the wetted wall. The bottom of the liquid droplet also has a large pressure value due to gravity.

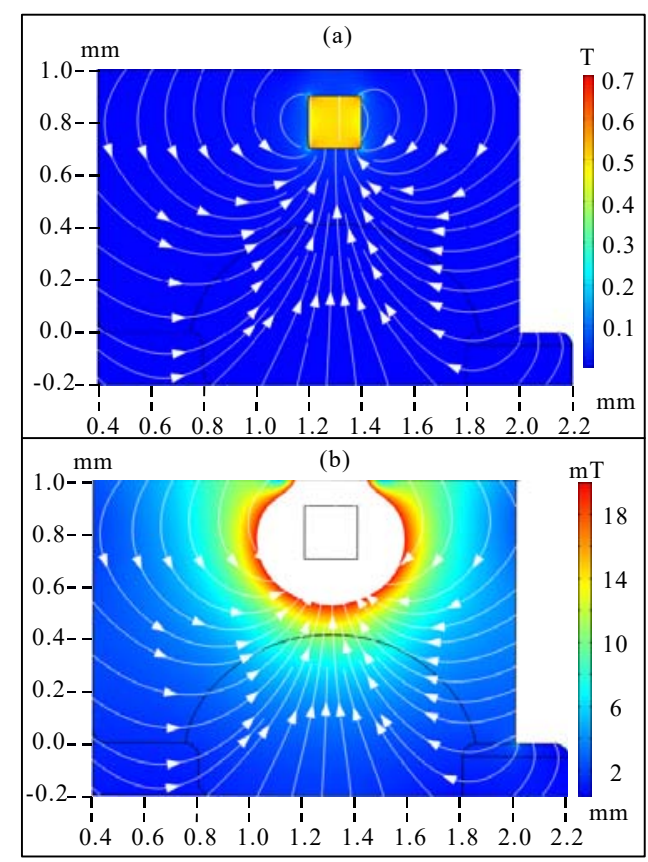

Fig. 4. At $\mathrm{t}=100 \mathrm{~ms} 、 V_{m}=16 \mathrm{~A}$, (a) the distribution of the whole magnetic field; (b) the distribution of the partial magnetic field $(\mathrm{B}<20 \mathrm{mT})$ 


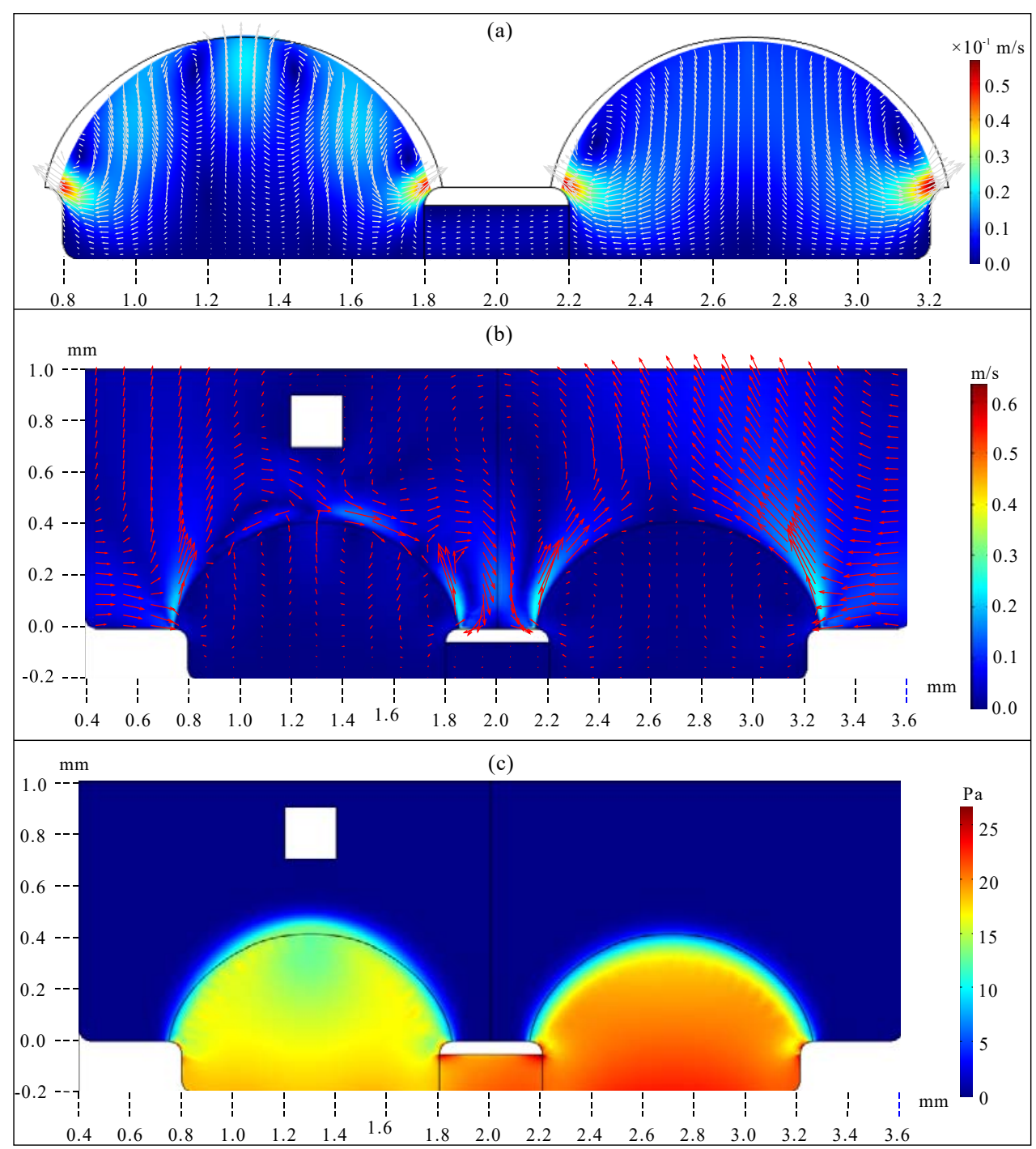

Fig. 5. At $V_{m}=16 \mathrm{~A}$, the distribution of flow field: (a) At $\mathrm{t}=4 \mathrm{~ms}$ and $\boldsymbol{\Phi}>0.6$, droplet velocity distribution; (b) At $\mathrm{t}=100 \mathrm{~ms}$, global velocity distribution; (c) At $\mathrm{t}=100 \mathrm{~ms}$, global pressure distribution.

The volume distribution of the droplet is shown in Fig 6, where the red domain indicates the droplet phase (DP), and the blue domain indicates air phase (CP). The color value between $0-1$ indicates the interface between the droplet and the air. At $V_{m}=4 \mathrm{~A}$, there is only a slight stretching phenomenon on the ferrofluid side droplet with contour close to the one only under gravity, as shown in Fig 6 (a). At $V_{m}=16 \mathrm{~A}$, the ferrofluid droplet is stretched upward while the lens droplet is stretched downward as shown in Fig 6 (a). Fig 6 (c) illustrates the interface of the droplets at $\boldsymbol{\Phi}=0.5$ under different magnetic potentials. The results reveal that the height of the ferrofluid droplet gradually increases while the lens droplet gradually decreases with the increasing of the magnetic potential. As shown in Fig 7, the area of the droplet in the two-dimensional model is considered to be conserved as the variation is very small which is around $1.1 \times 10^{-9} \mathrm{~mm}^{2}$. 


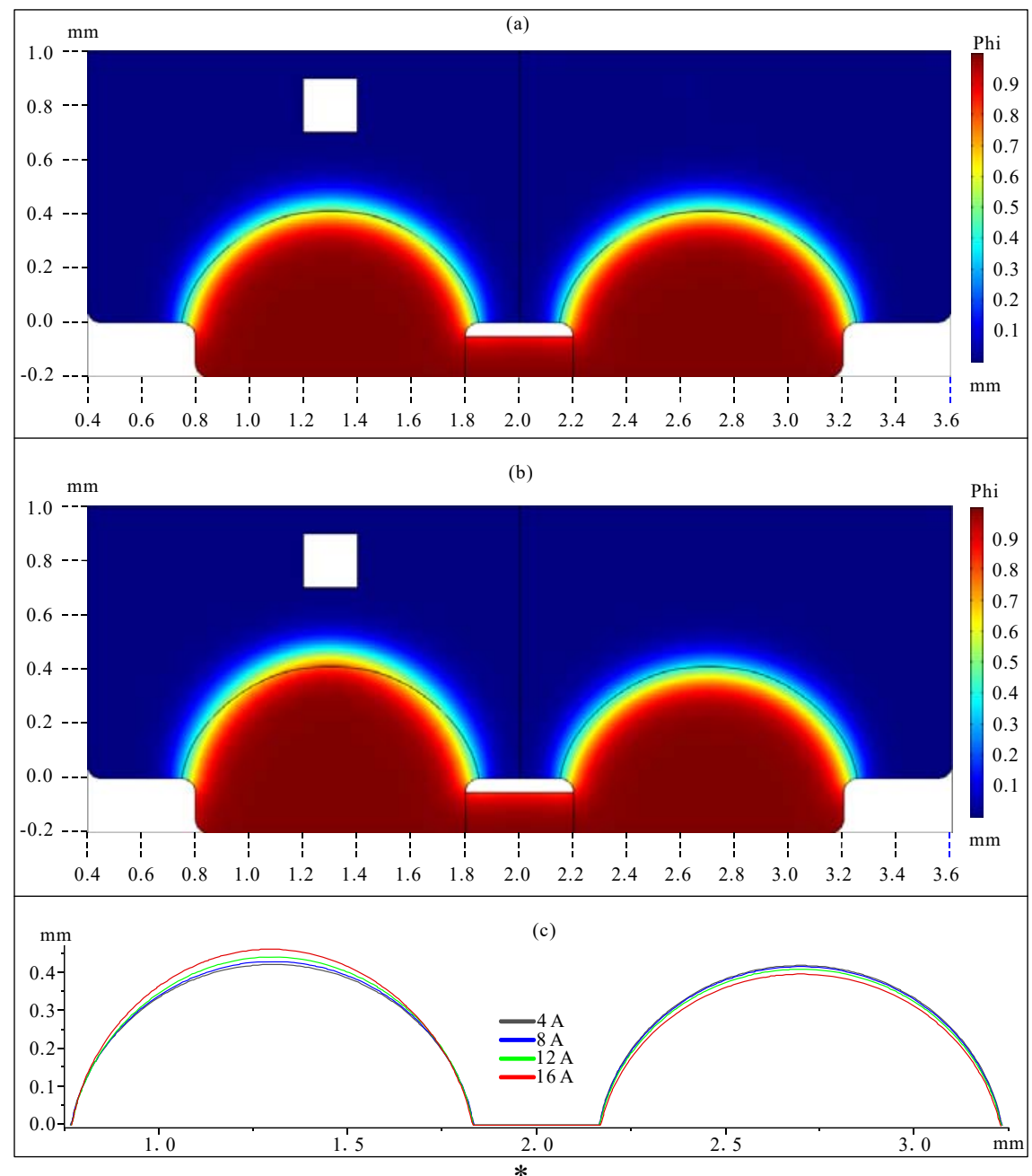

Fig. 6. At $\mathrm{t}=100 \mathrm{~ms}$, the distribution of droplet volume fraction: (a) At $V_{m}=4 \mathrm{~A}$, Droplet contour; (b) At $V_{m}=16$ A, Droplet contour; (c) At $V_{m}=4 \mathrm{~A}$ and $\boldsymbol{\Phi}=0.5$, Droplet interface.

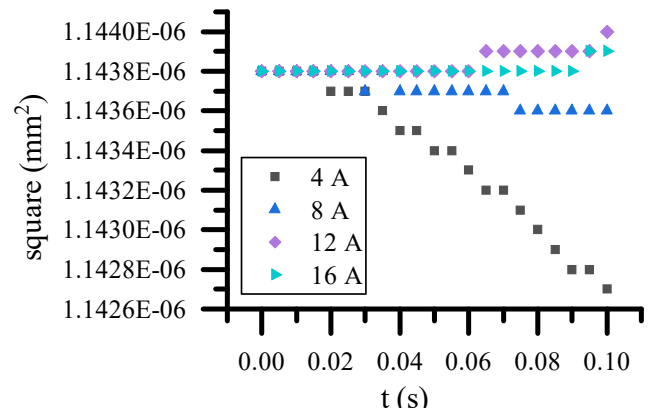

Fig. 7.The area of the droplet at different times and magnetic potential.

The results of optical module calculation are shown in Fig 8. Light is released from the incident boundary and reaches the bottom boundary of the computational domain after only $15 \mathrm{~ns}$. When there are only 8 incident rays, the inner and outer focal points of the droplets are inconsistent as shown in Fig 8 (a). This may be due to the calculation of the wetting wall, resulting in irregularities in the arc of the outer contour of the droplet. When the number of incident rays reaches 80 , the obvious focus of the rays can be observed, and only few of the rays outside the droplet deviates from the focus, as shown in Fig 8 (b).

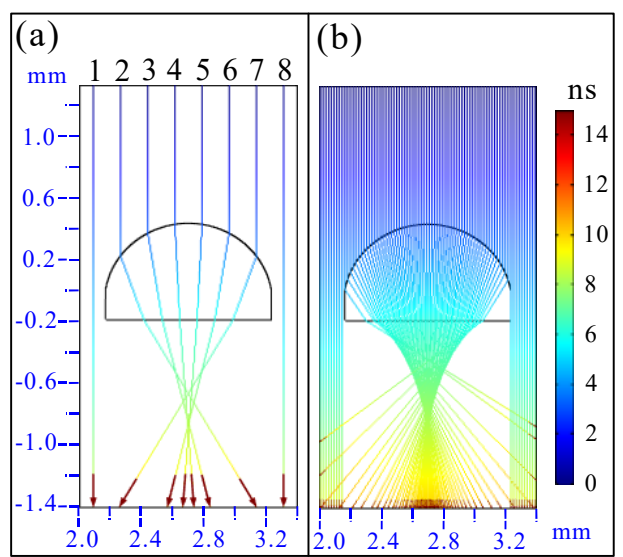

Fig. 8. At $V_{m}=4 \mathrm{~A}$, taking the lens droplet profile, ray optical calculation results: (a) The refraction of eight uniform incident light rays; (b) The refraction of 80 uniform incident rays

\section{Conclusion}

The magnetically actuated liquid lens, which has strong operability, can be flexibly zoomed by a magnetic field. In this work, the underlying physics of magnetically 
driven of ferrofluid droplet is systematically investigated and analyzed. The results are presented for flow field, magnetic field as well as pressure distribution for detailed information. The corresponding volume fraction of the ferrofluid droplet and lens droplet is discussed on the shape deformation and liquid flow through the intersection channel. The optical parameters of the lens droplet will be our futher focus for precise controlling and effective tuning under different magnetic field strength.

This work was partially supported by the Natural Science Foundation of Jiangsu Province (BK20160797), and the National Natural Science Foundation of China (11702134).

\section{References}

1. K.-H. Jeong, G. L. Liu, N. Chronis, L. P. Lee, Opt. Express 12, 2494-2500 (2004)

2. P. M. Moran et al. Appl. Phys. Lett. 88, 157 (2006)

3. Ren, H. \& Wu, S.-T. Opt. Express 15, 5931-5936 (2007).

4. N. Sugiura, S. Morita, Appl. Opt. 32, 4181-4186 (1993).

5. J. Chen, W. Wang, J. Fang, K. Varahramyan, J. Micromech. Microeng. 14, 675-680 (2004).

6. S. K. Cho, H. Moon, C. J. Kim. J. Microelectromech. S. 12, 70-80 (2003).

7. S. K.Fan, H. Yangb, W. Hsu, Lab Chip 11, 343-347 (2011).

8. J. Gong, C.-J. C. Kim, Lab Chip 8, 898-906 (2008).

9. U. C. Yi, C.-J. Kim, J. Micromech. Microeng. 16, 2053-2059 (2006).

10. C.-X. Liu, J. Park, J.-W. Choi, J. Micromech. Microeng. 18, 035023 (2008).
11. T. Krupenkin, S. Yang, P. Mach, Appl. Phys. Lett. 82, 316-318 (2003).

12. B. Berge, J. Peseux, Eur. Phys. J. E 3, 159-163 (2000).

13. A. F. Chrimes, K. J. Berean, A. Mitchell, G. Rosengarten, K. Kalantar-zadeh, Acs Appl. Mater. Inter. 8, acsami.5b10625 (2016).

14. C. G. Tsai et al. IEEE Photonics Tech. L. 21, 1396-1398 (2009).

15. S. Xu, Y. J. Lin, S. T. Wu, Opt. Express 17, 10499-10505 (2009).

16. A. Y. Malyuk, N. A. Ivanova, Appl. Phys. Lett. 112, 103701 (2018).

17. N. Chronis, G. L. Liu, K.-H. Jeong, L. P. Lee, Opt. Express 11, 2370-2378 (2003).

18. H. Yu, G. Zhou, F. S. Chau, S. K. Sinha, Sensors Actuat. A Phys. 167, 602-607 (2011).

19. H. Ren, D. Fox, P. A. Anderson, B. Wu, S. T. Wu, Opt. Express 14, 8031-8036 (2006).

20. N. Binh-Khiem, K. Matsumoto, I. Shimoyama, Appl. Phys. Lett. 93, 159 (2008).

21. S. H. Oh, K. Rhee, S. K. Chung, Sensors Actuat. A: Phys. 240, 153-159 (2016).

22. W. J. Xiao, S. Hardt, J. Micromech. Microeng. 20, 8 (2010).

23. B. A. Malouin, M. J. Vogel, J. D. Olles, L. L. Cheng, A. H. Hirsa, Lab Chip 11, 393-397 (2011).

24. H. C. Cheng, S. Xu, Y. F. Liu, S. Levi, S. T. Wu, Opt. Commun. 284, 2118-2121 (2011).

25. F M, C. I. White, Viscous Fluid Flow. (McGraw-Hill, 2006).

26. J. U. Brackbill, D. B. Kothe, C. Zemach, J. Comput. Phys. 100, 335-354 (1992). 